\title{
Utility of Serial Urinalyses and Urinary Cytology in the Evaluation of Patients with Microscopic Haematuria
}

\author{
Utilité d'une série d'analyses d'urine et de la cytologie urinaire dans l'évaluation des patients \\ ayant une hématurie microscopique
}

\author{
C. J. Rosser* ${ }^{*}$, K. Nakamura ${ }^{\dagger}$, J. Pendleton ${ }^{\dagger}$, S. Anai ${ }^{\dagger}$, M. Chang ${ }^{\ddagger}$, A. Kasraeian ${ }^{\S}$ L. Yeung ${ }^{\S}$
}

\begin{abstract}
BACKGROUND: Serial urinalyses have been advocated when haematuria is suspected.

OBJECTIVE: To determine the utility of serial urinalyses and urinary cytology in patients presenting for evaluation of microscopic haematuria.

METHODS: Eighty-five patients with the diagnosis of microscopic haematuria were evaluated at a tertiary-care hospital. All patients had a comprehensive urologic evaluation. Clinic and hospital records were reviewed for key factors (e.g., demographic, pathology, radiologic findings and operative findings).

RESULTS: One hundred ninety total urinalyses were reviewed. Eighty-eight (46\%) urinalyses were classified as normal, 87 (46\%) as haematuria ( $\geq 3 \mathrm{RBC} / \mathrm{hpf})$, and $15(8 \%)$ as pyuria/ bacteriuria. The initial urinalysis detected haematuria in $\mathbf{9 5 \%}$ of the patients. The addition of the second and third urinalyses detected haematuria in the remaining $5 \%$ of the patients with haematuria. Aetiologic factors for microscopic haematuria include urolithiasis $15(18 \%)$, infection $9(11 \%)$ and bladder lesion/tumor $6(7 \%)$. In this setting of microscopic haematuria, urinary cytology was not able to detect any of the five documented bladder tumors. Fifty-seven percent of patients had a negative haematuria evaluation.

CONCLUSION: In the evaluation of the patient with microscopic haematuria, serial urinalyses may have a low yield. Further prospective studies are needed to further evaluate serial urinalyses in this cohort. WAJM 2010; 29(6): 384-387.
\end{abstract}

Keywords: Urinalysis, haematuria, serial.

\section{RÉSUMÉ}

CONTEXTE: Les analyses d'urine en série ont été préconisées lorsqu'une hématurie est suspectée.

OBJECTIFS: Déterminer l'intérêt de l'analyse d'urine répétée et la cytologie urinaire chez les patients se présentant pour l'évaluation d'une hématurie microscopique.

METHODES: Quatre-vingt-cinq patients avec un diagnostic d'hématurie microscopique ont été inclus dans un hôpital de soins tertiaires. Tous les patients avaient une évaluation urologique complète. Les données essentielles ont été étudiées sur les dossiers de structures privées et hospitalières (par exemple les données démographiques, pathologiques, radiologiques et opératoires).

RESULTATS: Un total de 190 analyses d'urine ont été examinées. Quatre-vingt-huit (46\%) des analyses d'urine ont été classées comme normales, 87 (46\%) avaient une hématurie ( $\geq 3 \mathrm{RBC} / \mathrm{HPF})$ et 15 (8\%) comportaient une pyurie / bactériurie. L'analyse d'urine initiale détectait une hématurie chez $95 \%$ des patients. La répétition d'une deuxième et d'une troisième analyse d'urine décelait une hématurie chez les 5\% des patients restants atteints d'hématurie. Les facteurs étiologiques pour l'hématurie microscopique comprenaient 15 cas de lithiase urinaire (18\%), l'infection (11\%), ainsi qu'une lésion ou tumeur de la vessie dans cinq cas (7\%). Dans ce contexte d'hématurie microscopique, la cytologie urinaire n'a pas été en mesure de détecter l'une des cinq tumeurs de la vessie documentées. Cinquante-sept pour cent des patients avaient une recherche étiologique négative de l'hématurie.

CONCLUSION: Dans l'évaluation d'un patient pour hématurie microscopique, une série d'analyses d'urine peut avoir un faible rendement. Des études prospectives sont nécessaires pour évaluer plus en détail l'intérêt d'une série d'analyses d'urine dans ce groupe de patients. WAJM 2010; 29 (6): 384-387.

Mots-cles: Série d'analyses d'urine, hématurie microscopique, cytologie.

\footnotetext{
Division of Urology, The University of Florida, Jacksonville, Florida, Departments of Epidemiology and Health Policy Research and ${ }^{\S}$ Urology, The University of Florida, Gainesville, Florida.

*Correspondence: Dr. Charles J. Rosser, Department of Urology, University of Florida, College of Medicine, Suite N2-3, P.O. Box 100247, Gainessville, FL 32610. Phone: (352)273-7647, Fax (352) 392-8846. E-mail: charles.rosser@urology.ufl.edu.

Abbreviation: CT, Computed tomography; HPF, High power field; IVP, Intravenous pyelogram; RBC, Red blood cells; TNM, Tumor-NodeMetastasis.
} 


\section{INTRODUCTION}

Haematuria may be characterised as gross or microscopic in nature and may be caused by numerous factors (e.g., urolithiasis, haematologic abnormalities, infections, trauma, tuberculosis, and tumors). ${ }^{1}$ Some of these factors may be life threatening. Thus, prompt thorough evaluation and treatment are needed. Currently, evaluation for haematuria consists of inspecting the lower urinary tract by cystourethroscopy and upper tract evaluation with computed tomography scan of the abdomen and pelvis with intravenous contrast or intravenous pyelogram (IVP)., ${ }^{2,3}$

Recently, the definition of microscopic haematuria has changed to three or more red blood cells per highpower microscopic field in urinary sediment from at least two of three properly collected urinalysis specimens. ${ }^{4}$ Limited data are available to assess serial urinalyses in patients with a history of gross or microscopic haematuria. We hypothesise that serial urinalyses would add little to the evaluation of patients with haematuria, and that patients with documented haematuria should be extensively counseled about their risk of harboring a serious medical condition that may be causing the haematuria. Herein, we report findings of serial urinalyses and urinary cytology in patients presenting to a urology clinic for evaluation of microscopic haematuria.

\section{SUBJECTS, MATERIALS, AND METHODS \\ Study Population}

Institutional review board approval was obtained to query medical records for pertinent clinical information in patients referred by their primary care physician for evaluation of haematuria between January 2003 and July 2004 at Shands Jacksonville. This included 237 consecutive patients. Microscopic haematuria was defined as $\geq 3$ red blood cells (RBC) per high power field (HPF) in the urine. Initial evaluation included medical history, physical examination, and urinary cytology in select patients. Urine cultures were obtained in patients if there was a high suspicion that a urinary tract infection was the cause of the haematuria. Documented urinalysis was obtained in 85 patients, who formed our study cohort. Of these 85 patients, 54 and 31 patients had two and three urinalyses, respectively, available for evaluation. A total of 190 urinalyses were available for review. Median follow-up was 36 months (range 1-54 months).

\section{Cystoscopic Evaluation of the Lower Urinary Tract and Bladder Biopsy}

Haematuria evaluation included cystourethroscopy performed by an attending urologist with a 30- and 70degree endoscope in a $17-\mathrm{F}$ sheath in women in the outpatient setting, an 18-F flexible cystoscope in men in the outpatient setting, or with a 30- and 70degree endoscope with a $21-\mathrm{F}$ sheath in the operating room. All abnormal bladder lesions were biopsied and frank tumors were resected. Pathologic specimens were sent for evaluation in $10 \%$ formalin. In patients with atypia, suspicious cells, or carcinoma on urine cytology with no obvious bladder tumor visualized during cystoscopy, random bladder and prostatic biopsies (when applicable) were obtained.

\section{Radiologic Evaluation of the Upper Urinary Tract}

The majority of patients $(76 \%)$ had their upper urinary tracts evaluated by haematuria protocol computed tomography (CT) scan of the abdomen and pelvis. Haematuria protocol consisted of non-contrasted and contrasted images. ${ }^{5}$ Intravenous pyelograms (IVP) were used to evaluate the upper tracts of four patients. In patients with an intravenous contrast allergy or other contraindication for $\mathrm{CT}$ scan or IVP, magnetic resonance imaging $(n=3)$, or renal ultrasound in conjunction with retrograde pyelogram $(n=13)$ was used to evaluate the upper urinary tracts.

\section{Outcome Assessment}

Biopsy specimens were graded histologically according to standard grading system. ${ }^{6,7}$ The 2002 TumorNode-Metastasis (TNM) staging system was used for clinical staging. ${ }^{8}$ Clinic and hospital records were reviewed for several key factors, including urinalyses results, cystoscopic findings, radiologic findings, cytologic findings, tumor stage, and tumor grade.

\section{Statistical Analysis}

Differences in serial urinalyses detection rates were evaluated using the Chi-square test. $\mathrm{P}<0.05$ is significant. Statistical analysis was performed using SAS software (SAS Institute, Cary, North Carolina).

\section{RESULTS}

Of the 237 patients presenting for the evaluation of haematuria, 85 had documented microscopic haematuria and urinalyses for review and comprised our study group. Table 1 depicts the demographic and clinicopathologic characteristics of these 85 patients.

One hundred and ninety urinalyses were reviewed. Eighty-eight urinalyses were classified as normal (46\%), $87(46 \%)$ as haematuria ( $\geq 3 \mathrm{RBC} / \mathrm{hpf})$, and $15(8 \%)$ as pyuria/bacteriuria. Fifty-seven percent of patients were found to have a negative haematuria evaluation. The first urinalysis detected $95 \%$ of the patients with haematuria. The addition of second and third urinalyses detected haematuria

Table 1: Characteristics of 85 Patients with Microscope Haematuria

\begin{tabular}{|c|c|c|}
\hline Characteristic & No. & $\%$ \\
\hline \multicolumn{3}{|l|}{ Age } \\
\hline Median yrs & $2+13.3$ & \\
\hline Range yrs & $20-80$ & \\
\hline \multicolumn{3}{|l|}{$\operatorname{Sex}$} \\
\hline Male/Female & $39 / 46$ & $46 / 54$ \\
\hline \multicolumn{3}{|l|}{ Race } \\
\hline White & 48 & 56 \\
\hline Black & 28 & 33 \\
\hline Other & 9 & 11 \\
\hline Tobacco use & 43 & 51 \\
\hline Median pack yrs & 30 & \\
\hline \multicolumn{3}{|l|}{ Urinary Cytology } \\
\hline Normal & 65 & 76 \\
\hline Atypia & 20 & 24 \\
\hline Suspicious/Malignant & 0 & 0 \\
\hline \multicolumn{3}{|c|}{ Abnormality on radiologic evaluation* } \\
\hline Stone & 14 & 16 \\
\hline Renal Mass & 1 & 1 \\
\hline Other & 7 & 8 \\
\hline \multicolumn{3}{|l|}{ Cystoscopic findings* } \\
\hline Bladder stone & 2 & 2 \\
\hline Suspicious lesion/tumo1 & or 6 & 7 \\
\hline Other & 11 & 13 \\
\hline $\begin{array}{l}\text { Pathologic confirmed } \\
\text { malignancy }^{\dagger}\end{array}$ & 5 & 6 \\
\hline
\end{tabular}

*Patients may have had more than one cause of the haematuria; contains 2 patients with papillary neoplasm of low malignant potential. 
in an additional 4 (5\%) patients. One of these patients had a low grade, low stage transitional cell carcinoma and three had a negative evaluation.

Twenty (24\%) patients demonstrated atypia of whom only one was found to have transitional cell carcinoma of the bladder (clinical stage T1, grade 3 tumor). No patient demonstrated suspicious/ malignant cells on cytologic review. In addition, $19(22 \%)$ patients demonstrated an abnormality on cystoscopy, e.g., suspicious lesions/tumor $(n=6)$, bladder stone $(n=2)$ and other $(n=11), 22$ patients (26\%) demonstrated an abnormality on radiologic examination, and four patients (5\%) had abnormalities on both cystoscopic and radiologic review (e.g., $\mathrm{BPH}$ and/or bladder stone) that could be attributed to the haematuria. Eighty-three percent of the suspicious lesions/tumors proved to be cancer. Table 2 lists the causes of haematuria in the entire study cohort. The most common aaetiologic factors for microscopic haematuria, urolithiasis (18\%), infection (11\%) and bladder lesion/tumor (7\%) were most common.

Table 2: Aetiological Factors for Microscopic Haematuria*

\begin{tabular}{lc}
\hline Aetiology & N (\%) \\
\hline Renal calculi & $14(16)$ \\
Bladder tumor/lesion & $6(7)$ \\
Infection & $9(11)$ \\
Solid renal tumor & $1(1)$ \\
Bladder calculi & $2(2)$ \\
BPH & $5(6)$ \\
Trauma $^{\dagger}$ & $1(1)$ \\
Other $^{*}$ & $13(15)$ \\
\hline
\end{tabular}

*, may have more than one identified cause of haematuria ${ }^{\dagger}$, patient with Mitrofanoff and had difficulty catheterizing $\ddagger$, includes renal cyst $(n=7)$, urethral stricture $(n=5)$ and ureteral stricture $(n=1)$

\section{DISCUSSION}

Our study demonstrates that an overwhelming percentage of hematuric patients are detected with a single urinalysis. Only $5 \%$ of patients required serial urinalyses to detect haematuria. The incidence of a positive haematuria evaluation in patients is approximately 43\%. The most important reason for evaluating a patient with haematuria is to exclude a urologic neoplasm. The frequency of a malignancy causing the haematuria was $7 \%$. The most common causes of microscopic haematuria included urolithiasis, infection and bladder lesion/tumor. Over $95 \%$ of patients with urolithiasis and tumors demonstrated haematuria on their initial urinalysis. The one patient found to have a renal tumor had haematuria on initial urinalysis. Of the five pathologically proven bladder tumors, only one tumor defined as papillary neoplasm of low malignant potential had a negative initial urinalysis with subsequent positive urinalyses. In this setting of microscopic haematuria, urinary cytology was not able to detect any of the five documented bladder tumors. Thus in patients with microscopic haematuria, the utility of urinary cytology must come into question.

The aetiological factors for haematuria mirror those reported by Khadra and coworkers. ${ }^{9}$ Though microscopic haematuria seems less dramatic, its aaetiologic causes may range from insigni-ficant lesions to potentially life-threatening neoplastic lesions. ${ }^{10,11}$ Cost-effective screening for haematuria can be performed in an office setting by urine dipstick. ${ }^{12}$ Patients who screen positive on urinary dipstick must then proceed to urinary microscopic evaluation. ${ }^{13,14}$ This cannot be stressed enough since other solutes in the urine can interact with the components of a urinary dipstick to create a false positive result. Thus, patients with blood evident on dipstick must have this confirmed on microscopic analysis. Patients with documented haematuria ( $>3 \mathrm{RBC} / \mathrm{hpf}$ ) should then be referred for urologic evaluation. Thus meticulous attention to the proper initial evaluation of the hematuric patient is crucial.

Based on findings during urologic evaluation, imaging of upper urinary tracts, urine cytology, and lower urinary tract endoscopy may be required. Unfortunately, our current diagnostic capabilities will not allow us to evaluate patients for haematuria without performing these procedures. ${ }^{9}$ This is reflected by the current American Urological Association guidelines, which recommend that patients presenting with haematuria undergo a CT scan (haematuria protocol) and cystoscopy. ${ }^{2}$
The American Urologic Association recently convened a consensus panel that defined microscopic haematuria as three or more red blood cells per highpower microscopic field in urinary sediment from at least two of three properly collected urinalysis specimens. Unfortunately there is limited data to support the use of serial urinalyses. ${ }^{2}$ In our study, we demonstrated that serial urinalyses can detect an additional 5\% of patients with microscopic haematuria thus offering some utility. On the other hand, urinary cytology in this setting did not prove useful in detecting patients with bladder tumors. Other institutes may want to report on their experience with serial urinalyses and urinary cytology in patients with microscopic haematuria or a prospective study can be employed to determine their utility.

Our study has several limitations. First, this is a small, retrospective study from a single institution. Not only could biases have been introduced in patient selection and evaluation, this group may not represent patients with haematuria seen by urologists outside of a tertiary care setting or those seen by primary care physicians. Secondly, a paucity of outside medical records were available to review in order to determine how the patients initially were found to have haematuria (i.e., were they diagnosed based on history, urine dipstick, microscopic analysis). Lastly, there was no standardized follow-up protocol in place to monitor patients with a negative haematuria evaluation in order to determine possible long-term developments. In patients referred by primary care physicians for the evaluation of haematuria, the incidence of a positive haematuria evaluation was $43 \%$ at our institution. Serial urinalyses detected an additional $16 \%$ of patients with microscopic haematuria. The utility of serial urinalyses in the evaluation of the hematuric patient should be evaluated by others.

\section{REFERENCES}

1. Grossfeld GD, Carroll PR. Evaluation of asymptomatic microscopic haematuria. Urol Clin North Am. 1998; 25: 661-676. 